\title{
La influencia del entorno cultural en la eficiencia de las "mejores prácticas" en dirección de recursos humanos
}

\author{
The effect of cultural context on the efficiency of \\ best practices in human resource management
}

\author{
Aitziber Lertxundi LertXundi ${ }^{1}$ \\ JON LANDETA RODRÍGUEZ ${ }^{1}$ \\ Universidad del País Vasco (UPV-EHU) (España)
}

Recibido el 10 de marzo de 2010 y aceptado el 9 de octubre de 2010

$\mathrm{N}^{\mathrm{o}}$ de clasificación JEL: M12, F23, M21

DOI: $10.5295 /$ cdg.100213al

\section{Resumen:}

La eficiencia de los llamados Sistemas de Trabajo de Alto Rendimiento ha sido un objeto de estudio preferente en las dos últimas décadas. El análisis de su comportamiento se ha realizado considerando diversos factores que supuestamente moderan su eficiencia, entre los que destacan los factores internos de la empresa. Sin embargo, los factores externos, y más concretamente la perspectiva contextual, en contadas ocasiones han sido objeto de análisis. En este sentido, en este trabajo incorporamos el contexto cultural como variable moderadora de la eficiencia de la utilización de los Sistemas de Trabajo de Alto Rendimiento. Los resultados obtenidos a partir de una muestra de filiales exteriores de empresas multinacionales españolas, ponen de relieve la necesidad de tener en cuenta esta variable en las futuras investigaciones.

Palabras clave:

Mejores prácticas, HPWS, recursos humanos, contexto cultural, empresas multinacionales, España.

\begin{abstract}
:
The efficiency of High Performance Work Systems has been a special object of study for the last two decades. Analysis of their behaviour has involved the consideration of Several factors that supposedly moderate their efficiency, among which internal company factors stand out. However, contextual perspective has not been considered in great detail. In this regard, we go about our analysis incorporating cultural context as a possible moderating variable. From a sample of foreign affiliates of Spanish multinational companies, the results recommend that this variable be more closely considered in future investigations.
\end{abstract}

Keywords:

Best practices, HPWS, human resources, cultural context, multinational enterprises, Spain.

\footnotetext{
${ }^{1}$ Universidad del País Vasco (UPV-EHU), Departamento de Economía Financiera II, Facultad de Ciencias Económicas y Empresariales, Plaza Oñati 1, 20008 Donostia-San Sebastián. Email: aitziber.lertxundi@ehu.es jon.landeta@ehu.es
} 


\section{INTRODUCCIÓN}

La relación entre la dirección de los recursos humanos y el rendimiento organizacional ha sido un objeto de estudio preferente en el área de la Dirección Estratégica de Recursos Humanos (DERH). Si bien existe consenso sobre el efecto positivo que sobre los resultados empresariales tiene una dirección de recursos humanos (DRH) avanzada, continua todavía el debate en torno a las variables contingentes que podrían alterar la intensidad o la dirección de dicha relación. En este sentido, el contexto cultural es una variable de carácter contextual que, a priori, puede tener una influencia relevante sobre el binomio DRH-Rendimiento.

El paradigma universalista apunta que ciertas prácticas de recursos humanos, a las que se les denomina genéricamente "mejores prácticas", poseen un impacto positivo en todas las organizaciones, independientemente del tamaño, el sector o el país (Pfeffer, 1998). Por el contrario, bajo el paradigma contextual se pone de manifiesto la capacidad de definición y de condicionamiento que posee el conjunto de elementos institucionales y culturales de un país sobre las prácticas de DRH. El Enfoque Cultural aborda esta cuestión desde el punto de vista de lo que suponen las diferencias en los valores y costumbres sociales (Adler, 1997; Hofstede, 1984, 2001; Trompenaars, 1994). Este factor parece ser relevante tanto en la configuración de la DRH en las filiales de una empresa multinacional como en su aplicación en los contextos multiculturales (Bae et al., 1998; Tayeb, 2005; Yuen y Kee, 1993).

Esta línea de investigación en torno a las “mejores prácticas” en DRH ha sido fértil en estos últimos años, pero todavía está necesitada de nuevas evidencias empíricas. Algunos autores reprochan la escasa consideración recibida del contexto exterior a la organización y propugnan la ampliación del análisis para la incorporación de factores externos contextuales (Björkman y Xiucheng, 2002; Boselie et al., 2001). Por ejemplo, Appelbaum et al. (2000) señalan que la aplicación de los Sistemas de Trabajo de Alto Rendimiento (STAR), está estrechamente condicionada por el entorno regulativo. Se sugiere que el contexto social de las organizaciones trae consigo nuevos puntos de vista que ayudan a ampliar el conocimiento sobre la relación entre los sistemas de DRH y la eficiencia organizacional. Salvo algunas excepciones (Ahmad y Schroeder, 2003; Bae et al., 2003; Björkman y Xiucheng, 2002; Black, 1999; Park et al, 2003), es deficitaria la consideración que ha tenido el macrosistema social en esta literatura.

Por otra parte, los escasos trabajos empíricos que sí han abordado la efectividad de las "mejores prácticas" o STAR ${ }^{1}$ desde el punto de vista cultural e internacional no muestran unos resultados concluyentes. Ahmad y Schroeder (2003) estudiaron la generalización de la eficiencia de las siete prácticas propuestas por Pfeffer (1998) en distintos países, afirmando que, en general, un sistema de DRH “ideal” es más eficiente. Bae et al. (2003) analizaron el impacto de los STAR en un conjunto de compañías filiales y subsidiarias, concluyendo que éstos contribuían positivamente al rendimiento y destacando que este impacto era mayor en las locales, probablemente por su mejor conocimiento del entorno local. Björkman y Xiucheng (2002) hicieron lo propio con 62 filiales de empresas multina-

\footnotetext{
${ }^{1}$ Uno de los términos más empleados es el denominado High Performance Work Practices, propuesto por el Departamento de Trabajo de EE.UU.: U.S. Department of Labor (1993): High performance work practices and firm performance. Washington, DC: U.S. Government Printing Office. No obstante, también es frecuente el término High Performance Work Systems, que hace alusión a un nivel de análisis donde el objeto de estudio es el conjunto o el sistema de prácticas, en vez de las prácticas individuales.
} 
cionales de diversos países situadas en China y confirmaron la existencia de una relación positiva, a la vez que sugerían que el contexto institucional-cultural podría ejercer un efecto moderador. Por último, Park et al. (2003) no encontraron evidencias de que el contexto influyera en la relación causal.

Centrándonos en el área de estudio de las “mejores prácticas” en DRH, en este trabajo analizamos su efectividad sobre el rendimiento de la empresa, así como su interacción con el contexto cultural nacional donde se aplican. Ampliamos, por tanto, el análisis de las "mejores prácticas" al ámbito internacional, completando análisis anteriores, centrados preferentemente en el ámbito doméstico, con nuevas evidencias extraidas de contextos culturales diferentes.

\section{FUNDAMENTOS TEÓRICOS E HIPÓTESIS DE TRABAJO}

En el área de la DERH, la mayoría de las investigaciones en torno a la relación entre la DRH y el rendimiento coinciden en confirmar el impacto positivo que determinadas prácticas de DRH ejercen en los resultados empresariales. Esta relación se ha estudiado desde diversas perspectivas teóricas.

La perspectiva universalista asume la existencia de determinadas prácticas que son universalmente aplicables y competitivas, al margen de circunstancias internas o externas. Estas prácticas se conocen de forma genérica como "mejores prácticas", aunque de acuerdo al enfoque adoptado, se han utilizado etiquetas como "human capital-enhancing" (Youndt et al., 1996), "sistemas de trabajo de alto rendimiento" (Appelbaum et al, 2000) o "high involvement" (Guthrie, 2001) para referirse al mismo tópico de estudio. Esta perspectiva no plantea ningún tipo de interacción sinérgica entre ellas, sino que se consideran de forma aditiva. Entre los estudios empíricos que apoyan la universalidad se encuentran, entre otros, el de Arthur (1994), Camelo et al. (2004), Delaney y Huselid (1996), Guthrie (2001), Guthrie et al. (2009), Huselid (1995) y Koch y MacGrath (1996). Es reseñable que algunos de estos trabajos no muestran un apoyo pleno. Incluso algunos autores rechazan la universalidad (Capelli y Neuman, 2001; Godard, 2004; Purcell, 1999), mientras que otros recomiendan cautela o la aceptan con limitaciones (Gooderham et al., 2008; Guest et al., 2003; Rodríguez y Ventura, 2003; Wood y Menezes, 1998). En cuanto al nivel de análisis, destacan dos aproximaciones básicas diferenciadas. Por un lado, se encuentran trabajos que tratan sobre prácticas aisladas. En concreto, se han abordado aspectos como la compensación variable (Gerhart y Milkovich, 1990), el reclutamiento y la selección (Tepstra y Rozell, 1993), la evaluación del rendimiento (Borman, 1991) y la formación (Rusell, Terborg y Powers, 1985). Y por otro, trabajos que analizan un conjunto o sistema constituido por este tipo de prácticas (Arthur, 1994; Huselid, 1995; Ichniowski et al., 1997; MacDuffie, 1995; Walton, 1985), que vienen a ser los que se denominan STAR. Aunque en este último caso se considera un conjunto de prácticas, cabe señalar que en la perspectiva universalista no se plantea ningún tipo de interacción sinérgica entre ellas, sino que se consideran de forma meramente aditiva.

Por consiguiente, a pesar de que esta perspectiva cuente con un apoyo notable, existen estudios que reflejan cierta disparidad de resultados, lo que hace que siga siendo necesaria la aportación de nuevas evidencias empíricas sobre este objeto de estudio. 
Por lo tanto, de acuerdo con este enfoque universalista, la hipótesis que formulamos establece que la utilización de las “mejores prácticas" o, desde un punto de vista de conjunto, de los STAR, se relacionan positivamente con el rendimiento:

Hipótesis primera $\left(\mathrm{H}_{1}\right)$ : El grado de utilización de los STAR influye positivamente en el rendimiento.

Por su parte, desde la perspectiva contingente se parte de la premisa de que la validez y la aplicación eficaz de las prácticas no son generalizables a todas las circunstancias. En las relaciones con los resultados intervienen variables que alteran los efectos originales, por lo que deben ser consistentes con las prácticas. Se asume la existencia de interacciones y se establece que la relación entre la variable dependiente y las independientes relevantes será diferente para distintos niveles de la variable de contingencia crítica. Se incorpora en el análisis el concepto de ajuste. Sin embargo, algunos trabajos no apoyan este enfoque (Peck, 1994) y otros lo hacen de forma parcial (Fey y Björkman, 2001; Sanz-Valle et al., 1999). Yendo más allá, algunos autores incluso señalan que es escasa la evidencia que demuestra que el ajuste sea necesario o beneficioso (Delery, 1998). Con una orientación más configuracional, algunos trabajos recomiendan preferentemente el uso de conjuntos o sistemas de prácticas consistentes entre sí, y no tanto de prácticas aisladas (Appelbaum et al. 2000; Arthur, 1994; Hoque, 1999; Ichniowski et al., 1997; Koch y McGrath, 1996; MacDuffie, 1995). No obstante, algunos de los trabajos más respetados aconsejan actuar con prudencia en lo que concierne a esta complementariedad (Delaney y Huselid, 1996; Huselid, 1995) e incluso hay quienes afirman no haber encontrado en su estudio evidencia alguna de sinergias (Cappelli y Neumark, 2001).

Entre los factores externos que pueden condicionar la relación DRH-Rendimiento, los estudios comparativos señalan a la cultura y a las instituciones nacionales como dos de los factores que intervienen en el grado de adopción y difusión de las prácticas organizacionales en general (Laurent, 1986; Myloni et al., 2004; Schuler y Rogovsky, 1998) y supuestamente, de los sistemas de dirección de alto compromiso o de trabajo de alto rendimiento (Black, 1999; Bae y Rowley, 2001; Martin y Beaumont, 1998). Desde el enfoque cultural se apunta hacia la necesidad de que el entramado institucional y cultural de un país sea incorporado como un elemento más de análisis en la implementación de las “mejores prácticas" puesto que, aunque éstas actúen en cualquier país, sector o sociedad sobre elementos vinculados con la habilidad, la motivación y la oportunidad de los trabajadores, el contexto cultural puede influir en la combinación de las políticas y prácticas que los conforman. En este sentido, se apunta que, pese a los hallazgos sobre los efectos de prácticas de recursos humanos en el desempeño organizacional, la efectividad de las mejores prácticas en las compañías no occidentales no es todavía clara (Rhodes et al., 2008). El hecho de pasar por alto la vinculación existente entre el contexto y las prácticas de DRH puede ser causa de conflictos e ineficiencias (Bae et al., 1998; Gill y Wong, 1998; Kim y Gray, 2005; Tayeb, 2005; Verburg et al., 1999; Yuen y Kee, 1993). Desde una visión más optimista, Newman y Nollen (1996) aportan evidencias de que la congruencia entre la cultura nacional y las prácticas de gestión posee efectos positivos en los resultados. No obstante, estos mismos autores relatan las dificultades asociadas a los STAR, cuando, por ejemplo, señalan que la retribución contingente o la participación de los empleados, dos de sus características propias, no disfrutan del mismo éxito en todos los países. 
En este sentido, la literatura ya ha encontrado algunas evidencias (escasas) que asocian positiva o negativamente la eficiencia de determinadas prácticas con algunas características o dimensiones culturales. En efecto, tomando como referente teórico el conocido modelo de Hofstede $^{2}(1984,2001)$ la variable cultural se puede descomponer en cuatro dimensiones. La dimensión individualismo hace referencia al grado en que una sociedad valora los objetivos, la autonomía y la intimidad personal en contraposición a la lealtad hacia el grupo, el respeto hacia sus normas, la participación en actividades colectivas, la cohesión social y el alto grado de socialización. Por ejemplo la retribución ligada al rendimiento está más relacionada con el individualismo alto (Gómez-Mejía, Balkin y Cardy, 1997). Desde el punto de vista de la autonomía, una de las características de los equipo de trabajo en los STAR, es de esperar que cuanto más individualista sea un país mayor será la disposición de las empresa a delegar más autonomía a cada uno sus empleados a cambio de ser más exigente con los resultados que alcancen éstos. Un individualismo bajo, o en otras palabras, el colectivismo, se ha mostrado que está asociado con más cooperación y actitudes positivas hacia los equipos, por lo que se estima que los trabajadores de una sociedad en la que predomine este valor accedan más fácilmente a incorporar el trabajo en equipo (Eby et al., 2000; Kirkman y Shapiro, 2001; Steensma et al., 2000), algo que supuestamente favorecería la implantación de los STAR. Sin embargo, estas mismas personas pueden mostrar reticencias ante la incorporación de otros procesos, técnicas o, en general, cualquier factor organizacional que demande ciertos rasgos individualistas (Bae y Lawler, 2000), como resulta ser la remuneración incentivada individual. En conjunto, podríamos establecer que un alto grado de individualismo podría ser un contexto favorable para la eficiencia de estos sistemas.

Hipótesis segunda (a): Un alto grado de individualismo ejerce un efecto moderador positivo en la relación entre el grado de uso de los STAR y el rendimiento.

La distancia al poder se refiere al grado de aceptación y respeto de la distribución desigual del poder por parte de los miembros menos favorecidos de las organizaciones, las instituciones y la sociedad. Se ha podido observar que, por ejemplo, en lo que respecta a la participación de los empleados, su eficacia es cuestionable en aquellos países con una alta distancia al poder, con tintes jerárquicos y paternalistas muy marcados, dado el temor y la desconfianza de los empleados a actuar con iniciativa propia (Adler, 1997). Así mismo, en las culturas muy jerárquicas el diseño formal de la organización tiende a la mayor concentración de poder y, en general, estas sociedades se muestran reacias a la implementación de equipos autodirigidos (Aycan et al., 2000), uno de los pilares de de los STAR. El carácter jerárquico de las culturas asiáticas, por ejemplo, en donde los individuos de estatus inferior tienden de forma natural a mostrar "respeto" hacia aquellos de estatus más alto y éstos, a su vez, esperan esta actitud hacia ellos, parece que constituyen un escenario poco favorable hacia la eficiencia de estos sistemas. Los países nórdicos, por el contrario, presentan distancias al poder mucho más reducidas, lo que puede facilitar la comunicación, participación y confianza y, en suma, aceptar de mejor grado y con mayor aprovechamiento la utilización de los STAR.

\footnotetext{
${ }^{2}$ El modelo de Hofstede identifica cualquier contexto cultural nacional a partir de cuatro dimensiones principales: individualismo-colectivismo, distancia al poder, aversión a la incertidumbre y masculinidad-feminidad. Este modelo asigna a los países una puntuación de 1 a 100 en cada una de estas dimensiones.
} 
Por tanto, nuestra hipótesis es que la interacción de esta dimensión cultural con la aplicación eficiente de los STAR es negativa.

Hipótesis segunda (b): Un alto grado de distancia al poder ejerce un efecto moderador negativo en la relación entre el grado de uso de los STAR y el rendimiento.

La aversión a la incertidumbre trata del grado en que los individuos se sienten amenazados frente a situaciones desconocidas o inciertas y para las que desarrolla creencias o sistemas que tratan de evitarlas. Una sociedad muy adversa al riesgo tiende a trabajar con actividades más estructuradas. En entornos de estas características cabe pensar que la actitud de reducir la incertidumbre llevará al empresario a conceder menos autonomía a los trabajadores, así como a implantar menos equipos autónomos de trabajo, al mismo tiempo que los propios trabajadores se sentirán más confortables con tareas lo más estructuradas y predecibles posibles. Igualmente, un entorno muy adverso a la incertidumbre tampoco sería favorable hacia inversiones considerables en la formación de sus empleados, debido a la falta de certeza en cuanto a la reversión de beneficios de esta actividad. En cuanto a la retribución contingente, al tratarse de un elemento que introduce variabilidad e incertidumbre, tanto desde el punto de vista de los empleados y como del empresario, posiblemente sea de difícil aceptación cultural.

En conjunto, nuestra hipótesis es que el efecto moderador de esta dimensión cultural sobre la utilización de los STAR sobre los resultados empresariales será negativo.

Hipótesis segunda (c): Un alto grado de aversión a la incertidumbre ejerce un efecto moderador negativo en la relación entre el grado de uso de los STAR y el rendimiento.

En lo que respecta a la dimensión de masculinidad, en las sociedades masculinas los valores predominantes son la competencia y el éxito profesional y económico, mientras que en las sociedades femeninas lo son el consenso e interés por los demás y la calidad de vida, entre otros. En las sociedades masculinas se pone énfasis en los resultados y en la competitividad. Se trata de recompensar a cada uno de acuerdo con su rendimiento (Hofstede, 1998). En este sentido, la "masculinidad" facilitaría la implantación y aceptación de los incentivos por desempeño. Además, la predominancia de valores como la competitividad o el éxito podrían contribuir a la implantación eficiente de otras prácticas que conforman los STAR. Por ejemplo, la participación y asunción de responsabilidades, como forma de desarrollo y competición personal, orientada a la promoción y a la mejora salarial.

Por consiguiente, en culturas "masculinas" presumiblemente la aplicación de STAR confiere a los miembros de la empresa mayores posibilidades para hacer mejor sus tareas y destacar respecto a sus compañeros, por lo que la hipótesis que establecemos en relación a esta dimensión es:

Hipótesis segunda (d): Un alto grado de masculinidad ejerce un efecto moderador positivo en la relación entre el grado de uso de los STAR y el rendimiento.

Las cuatro subhipótesis conducen a la siguiente hipótesis general sobre el efecto moderador del contexto cultural en la relación entre la utilización de las STAR y el rendimiento empresarial:

Hipótesis segunda $\left(\mathrm{H}_{2}\right)$ : El contexto cultural ejerce un efecto moderador en la relación entre el grado de uso de las STAR y el rendimiento. 
Figura 1

Variables de análisis e hipótesis

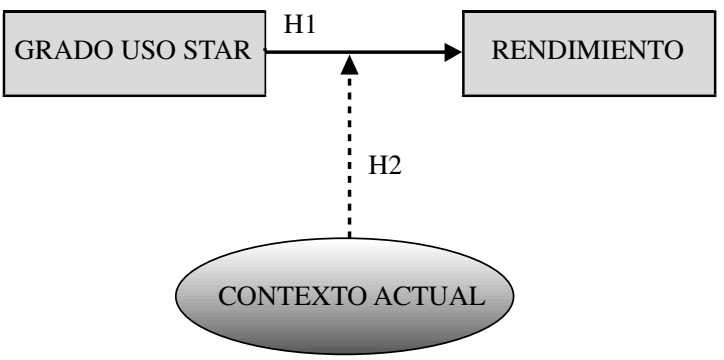

\section{METODOLOGÍA}

Para el contraste de las hipótesis formuladas optamos por acotar una población de empresas a las que su acceso fuera posible, que pertenecieran a entornos culturales distintos, pero que tuvieran un grado de homogeneidad suficiente como para poder identificar, en su caso, la influencia de los STAR y de la cultura nacional sobre los resultados de la organización. Optamos por empresas que fueran filiales productivas de firmas españolas, radicadas en el extranjero y con más de 100 trabajadores. El marco muestral se extrajo de diferentes bases de datos y se contrastó a través de la revisión de las webs corporativas y de consultas telefónicas. El número total de empresas identificadas que cumplían las condiciones establecidas fue de 256. La técnica de recogida de información se basó en la entrevista personal o encuesta telefónica, al director-gerente o al director de recursos humanos, en su caso ${ }^{3}$. La muestra finalmente resultante está integrada por 58 filiales exteriores productivas del sector manufacturero dispersas por distintos países (29 Europa, 16 Latinoamérica, 7 Asia y 6 EEUU) (tasa de respuesta, 22\%). Dado el tamaño de la muestra, para un nivel de confianza del $95 \%$ y el caso más desfavorable en lo que respecta a la varianza de la población, el error muestral se eleva al $11 \%$.

\footnotetext{
${ }^{3}$ En primer lugar nos dirigíamos al responsable de RRHH corporativo. Sin embargo, en algunas empresas la única persona que disponía de la información que solicitábamos era el director general y, por tanto, era ésta la persona más indicada para entrevistar. Hubiera sido deseable que el cargo de todas las personas que respondieron hubiera sido el mismo, a fin de evitar posibles sesgos, pero la realidad de cada empresa hacía imposible alcanzar ese ideal. En cualquier caso, las personas que respondieron a la entrevista tenían la suficiente visión global de la empresa y de sus filiales, y de sus prácticas de gestión de recursos humanos (se invalidaron las encuestas incompletas), por lo que entendemos que el riesgo de un sesgo significativo es reducido. A este respecto, Huselid y Becker (2000) afirman que en muchas ocasiones son el director de personal o el propio gerente los mejor situados o quizás los únicos cualificados para proporcionar la información requerida para numerosos puestos de trabajo. Persiste el debate en torno a la fuente válida de información en recursos humanos. No obstante, buena parte de los estudios empíricos tiende al uso de una única persona y casi la mitad confía en el responsable de recursos humanos (Boselie et al,. 2005). (Huselid, M.A. y Becker, B.E. (2000): "Comment on measurement error in research on human resources and firm performance: how much error is there and how does it influence effect size estimates?", Personnel Psychology, vol. 53, $\mathrm{n}^{\circ} .4$, pp. 835-854. / Boselie, P., Dietz, G. y Boon, C. (2005): "Commonalities and contradictions in HRM and performance research”, Human Resource Management Journal, vol. 15, n’.3, pp. 67-94.)
} 


\subsection{Operacionalización de las variables}

\section{A. Variables independientes}

(a) Grado de uso de los STAR. Recoge la intensidad con la que se utilizan en la empresa las prácticas de recursos humanos consideradas más eficientes en la literatura. Existen diversos enfoques para operacionalizar el grado de uso de las "mejores prácticas" o, desde la perspectiva de conjunto, los STAR, y entre ellos, optamos por el procedimiento de asignar las prácticas a conjuntos en base a los preceptos teóricos, con la posterior verificación de la fiabilidad de las escalas (Guthrie, 2001; MacDuffie, 1995; Yound et al.,1996; Way, 2002). Además, decidimos utilizar una medida única para su medición, en consonancia con los argumentos propuestos por Barney y Wright (1998) y Becker et al. (1997), quienes aconsejan la utilización de un índice unitario que de cuenta del empleo de estas prácticas. Así proceden también otros autores (Appelbaum et al., 2000; Arthur, 1994; Chang, 2006; Guest et al., 2003; Guthrie et al., 2009; Ichniowski et al., 1997; MacDuffie, 1995; Park et al., 2003; Youndt et al., 1996). La puntuación obtenida por cada empresa en dicho índice representaría el grado de uso que se hace de las prácticas contenidas en un sistema de este tipo. Por su parte, en línea con los trabajos de Appelbaum et al. (2000), Delaney y Huselid (1996) y Guest (1997), recurrimos al enfoque teórico AMO (Ability, Motivation, Opportunity) como base que permite definir y estructurar el conjunto de las prácticas integrantes de un STAR (Appelbaum et al., 2000; Boxall y Purcell, 2003). Este enfoque afirma que la efectividad de los STAR precisa del cumplimiento de tres componentes básicos: la habilidad de los trabajadores, su motivación y un entorno de trabajo que provea del apoyo necesario y la oportunidad para expresarse. Este modelo tiene como núcleo las políticas de formación y selección que garanticen una fuerza de trabajo con una apropiada cualificación, la existencia de incentivos adecuados, así como la oportunidad para una significativa participación en las decisiones. Las políticas y prácticas de recursos humanos jugarían su papel en la influencia que ejercen en las cualificaciones, en la motivación y en las oportunidades proporcionadas, de modo que éstos, a su vez, inciden positivamente en resultados intermedios como la confianza, el compromiso organizacional y en la satisfacción en el trabajo. A partir de esta concepción, la escala de medida que se utilizará se configura como una medida compuesta por seis dimensiones estructuradas en tres indicadores (figura 2). Las prácticas consideradas para su valoración se extraen de la revisión de los trabajos empíricos y teóricos más importantes pertenecientes a esta línea de investigación, incluyendo aquellas que con mayor frecuencia se consideran para la formación de estos sistemas (Arthur, 1994; Bae y Lawler, 2000; Delery y Doty, 1996; Delaney y Huselid, 1996; Guthrie, 2001; Huselid, 1995; Ichniowski et al., 1997; Peck, 1994; Pfeffer, 1998).

Tabla 1

\section{Prácticas consideradas en la investigación}

\section{Formación}

Volumen de inversión destinado a actividades de formación, así como la existencia de programas formales de formación de diverso tipo (resolución de problemas, habilidades técnicas...). 


\section{Selección}

Prácticas de selección rigurosas, basadas en criterios objetivos, diseñadas para cada puesto de trabajo y dirigidas a garantizar que el candidato seleccionado posee las cualificaciones necesarias para la labor a realizar.

\section{Comunicación}

Existencia de vías de comunicación que proporcionan la base que capacita a las personas para la adecuada coordinación y gestión. Existencia de mecanismos tales como, buzones de sugerencias, banco de aportaciones u otro tipo de canales para plantear ideas, quejas y aportaciones, en general.

\section{Retribución}

Prácticas de retribución de naturaleza contingente sobre la base de la productividad alcanzada por el empleado y el establecimiento de sistemas de evaluación de desempeño.

\section{Participación}

Acciones tendentes a favorecer la participación de los empleados en las decisiones y acciones, a fomentar la iniciativa y autonomía de los empleados en sus trabajos y que contribuyen a establecer una relación de cooperación y confianza

\section{Trabajo en equipo}

Existencia de grupos formales de trabajo autónomos de resolución de problemas, de calidad, de proyectos, etc.

El número de ítems propuestos fue de veinticuatro. Para la cuantificación de cada uno de los indicadores se calculó la media aritmética de los ítems que lo componen. La variable Índice STAR, se obtiene a partir de la suma de los seis grupos de indicadores, en línea con otros trabajos en este campo (Chang, 2006; MacDuffie, 1995; Park et al., 2003).

Figura 2

\section{Construcción y estructura del Índice STAR}

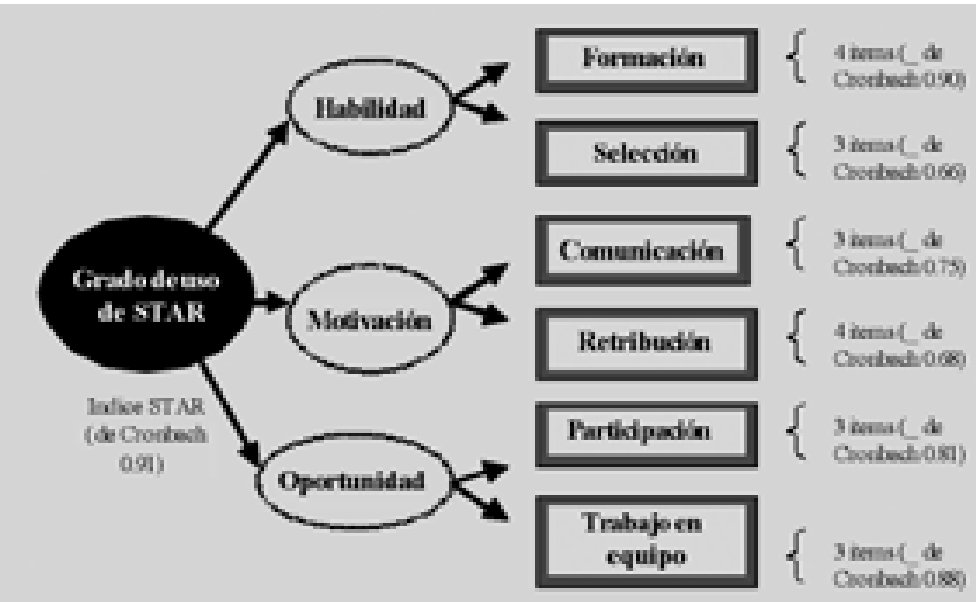

Nota: Items medidos mediante escalas Likert (1=no se utilizan - 7=se utilizan siempre) 
A fin de garantizar los aspectos básicos en la construcción de la escala se ha seguido el siguiente procedimiento (Hair et al., 1999). En primer lugar, la definición conceptual se ha llevado de tal forma que cumpliese la validez de contenido. Para el diseño del cuestionario inicial y la propuesta de los ítems nos basamos, en primer lugar, en una exhaustiva revisión de la literatura pertinente y, en segundo, en la revisión del cuestionario piloto por parte de diversos académicos y directivos de las empresas multinacionales. En segundo lugar, se ha llevado a cabo un análisis de fiabilidad basado en los coeficientes $\alpha$ de Cronbach de cada uno de los indicadores. Esto nos ha aconsejado eliminar cuatro de los ítems inicialmente planteados, con lo que el número final de ítems se fija en veinte. Seguidamente, se ha verificado la unidimensionalidad del índice mediante un Análisis Factorial de Componentes Principales. Como se esperaba, se ha extraído un único factor con un valor propio mayor que la unidad, lo que demuestra la unidimensionalidad del índice ${ }^{4}$. Por último, al objeto de validar la escala, se ha verificado la validez convergente del índice a través del cálculo de la correlación del mismo con un ítem que, con naturaleza claramente subjetiva, invitaba al encuestado a puntuar, en una escala de 1 al 5, (1=es peor; $5=$ es mucho mejor), la competitividad del sistema de DRH. La correlación de Pearson para dicho ítem y el Índice STAR para la matriz, de 0,708 , corrobora la validez convergente. Con todo ello, consideramos fiable y válida la escala de medición diseñada.

(b) Contexto cultural. Para su operacionalización se adopta el modelo propuesto por Hofstede (1984, 2001). Su aplicación ha sido muy amplia (Agarwal, 1994; Bonache y Cerviño, 1997; García y García, 2007; Gómez-Mejía y Palich, 1997; López, 2007; Morosini et al., 1998; Newman y Nollen, 1996; Schuler y Rogovsky, 1998). A pesar de no estar exento de detractores y críticas (Chiang, 2005; Gerhart y Fang, 2005; McSwweeney, 2002), este modelo se considera el más desarrollado sobre valores culturales (SØndergaard, 1994). Las dimensiones que propone señalan valores culturales universales, de modo que permite realizar comparaciones entre culturas nacionales (Mead, 1998) y permite cuantificar las diferencias culturales entre países, ya que prácticamente todos los países están ya medidos según las dimensiones de este modelo.

\section{B. Variable dependiente}

(a) Rendimiento. Se mide a partir de valoraciones subjetivas sobre diversos resultados economicos y financieros. Este tipo de medidas de percepción gozan de buena acogida entre los autores (Bae y Lawler, 2000; Delaney y Huselid, 1996; Fey y Björkman, 2001; Ngo et al., 1998; Yound et al., 1996). Sin embargo, el posible sesgo que puede haber entre ambas y el hecho de recurrir al responsable de recursos humanos o el gerente para la recogida de la información, conlleva una limitación que debe considerarse en la interpretación de los resultados. Se les indicaba a los encuestados que expresaran su grado de satisfacción en relación a la productividad de los empleados (ventas por empleado), los resultados financieros y los resultados globales alcanzados en promedio en los tres últimos años,

\footnotetext{
${ }^{4}$ Medida de adecuación muestral de Kaiser-Meyer-Olkin: 0,849; test de esfericidad de Bartlett: 255,896 (ChiCuadrado, Sig.: 0,000). Varianza total explicada: $56 \%$. Aunque es menor que el $60 \%$, este factor no va a sustituir a los seis indicadores, se obtiene exclusivamente a efectos de comprobación de la unidimensionalidad.
} 
en una escala tipo Likert de 7 puntos (1=satisfacción muy baja 7=satisfacción muy alta). El cálculo del valor de la variable Rendimiento se ha realizado como la media aritmética del valor en los tres ítems citados ( $\alpha$ de Cronbach: 0,940). Para la verificación de la unidimensionalidad de la escala compuesta, se ha llevado a cabo, una vez más, el Análisis de Componentes Principales ${ }^{5}$, donde se ha extraído un único factor (varianza total explicada: $89,404 \%)$.

\section{Variables de control}

Tamaño de la empresa. Lo medimos en su dimensión de tamaño de plantilla, a través del logaritmo del número de trabajadores (Guthrie, 2001; Huselid, 1995). En opinión de algunos autores, las grandes organizaciones pueden ser más proclives y tener más medios para la utilización de prácticas de recursos humanos más avanzadas (Jackson y Schuler, 1995, Youndt et al., 1996). Sin embargo, otros autores no han encontrado evidencias concluyentes de esta relación (Geary, 1999; Osterman, 1994). Por consiguiente, debido a su posible vinculación con la variable dependiente, decidimos introducir el tamaño de la empresa como variable de control.

\section{ANÁLISIS Y DISCUSIÓN DE RESULTADOS}

Para el contraste de las hipótesis recurrimos a dos técnicas diferentes: al análisis de regresión con términos de interacción y a las Pruebas t de diferencias de medias.

En relación con la primera de ellas, aplicamos el siguiente modelo de análisis

Rendimiento $=\mathrm{a}+\mathrm{b}_{1}$ *Índice STAR $+\mathrm{b}_{2}{ }^{*}$ Contex to Cultural $+\mathrm{b}_{3} *$ Índice STAR $*$ Contexto Cultural $+\varepsilon$

para cada una de las cuatro dimensiones culturales del modelo de Hofstede (Individualismo/Distancia al Poder/Aversión a la Incertidumbre/Masculinidad).

La construcción de una ecuación de regresión que incluya una interacción requiere que todos los términos de orden inferior deban ser introducidos (Aguinis, 1995; Cohen y Cohen, 1983). Siguiendo las recomendaciones de los autores (Aiken y West, 1991; Jaccard, Turrisi y Wan, 1990), antes de formar el término multiplicativo se procedió a centrar las variables para evitar los probables problemas de multicolinealidad entre éste y las variables que lo conforman. A continuación, se comprobó el cumplimiento de los supuestos básicos para la aplicación.

El modelo 2 constituye el modelo base sobre el que se añaden los efectos de interacción. Así, para el caso de Individualismo, los resultados globales se recogen en el modelo 4, para la Distancia al Poder en el modelo 6, para la Aversión a la Incertidumbre el modelo 8 y por último, en el caso de la Masculinidad en el modelo 10.

\footnotetext{
${ }^{5}$ Medida de adecuación muestral de Kaiser-Meyer-Olkin: 0,772; test de esfericidad de Bartlett: 304,717 (ChiCuadrado, Sig.: 0,000). Varianza total explicada: $89,404 \%$.
} 
Tabla 2

Resultados del análisis de regresión

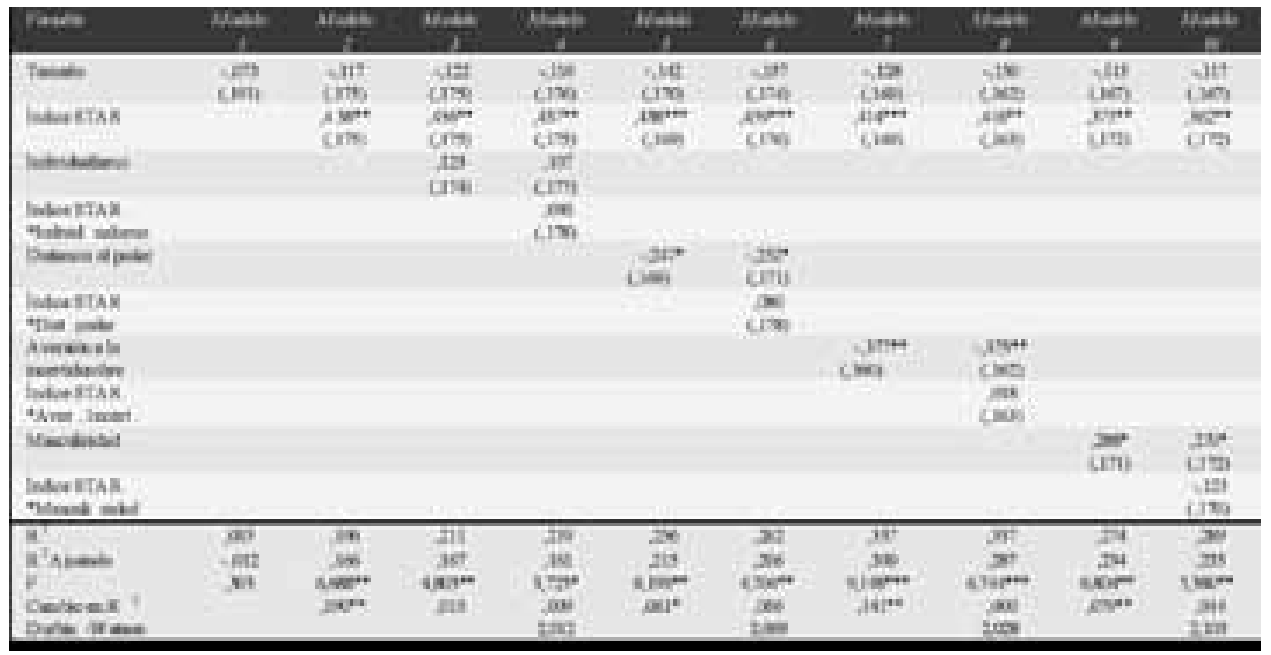

Variable dependiente: Rendimiento. Coeficientes de regresión estandarizados. Entre paréntesis: errores típicos de estimación $* \mathrm{p}<0.05 ; * * \mathrm{p}<0.01 ; * * * \mathrm{p}<0.001$

Se observa que en el modelo sin interacciones (modelo 2) la variable Índice STAR está positiva, moderada y significativamente relacionada con el rendimiento (Beta $=0,438$; Sig.=0,001). Este impacto se mantiene cuando introducimos los términos de interacción, aunque en el caso de la dimensión Masculinidad es sensiblemente inferior. Estos valores, nos indican que la utilización de los STAR afecta positivamente a los resultados, lo que nos lleva a aceptar la primera hipótesis. Este resultado es consistente con las evidencias empíricas aportadas por Arthur (1994), Camelo et al. (2004), Delaney y Huselid (1996), Guthrie (2001, 2009) y Koch y MacGrath (1996).

En el caso de los términos de interacción, para comprobar la existencia del efecto moderador, comparamos los valores de los coeficientes de determinación $\left(\mathrm{R}^{2}\right)$ correspondientes al modelo sin el efecto de interacción y al modelo con la interacción. El cambio producido en $\mathrm{R}^{2}$ no es estadísticamente significativo, como tampoco lo es, por definición, el coeficiente de regresión asociado al término de interacción $\left(b_{3}\right)$ para ninguna de las dimensiones culturales. A tenor de estos resultados, no se puede hablar de un efecto moderador del contexto cultural en la relación entre el grado de uso de los STAR y el rendimiento empresarial. Sin embargo, esto no significa necesariamente que no exista, puesto que este podría no ser lineal. Al introducir los términos de interacción en forma multiplicativa, estamos tratando solo una forma funcional, la lineal. Pero el efecto moderador puede adoptar otra forma funcional alternativa, de manera que el hecho de que no se obtengan resultados estadísticamente significativos con la incorporación de términos multiplicativos convencionales puede indicar la existencia de formas alternativas, más que la ausencia de efecto moderador (Jaccard et al., 1990). 
Por ello, completamos el análisis mediante las Pruebas $t$ de diferencias de medias, a fin de corroborar la ausencia de este efecto. En concreto, nos basamos en uno de los procedimientos utilizados en el trabajo de Newman y Nollen (1996), donde se analizaba el efecto de la congruencia entre las prácticas de gestión y el entorno cultural en el rendimiento empresarial -operacionalizado también el entorno cultural mediante el modelo cultural de Hofstede-.

Para poder proceder con el análisis, dividimos la muestra de empresas en grupos homogéneos con arreglo a su grado relativo de uso de los STAR y del nivel en la dimensión cultural donde se posiciona su país. La dicotomización de la variable Índice STAR lo efectuamos a partir de la mediana, de manera que la nueva variable dicotómica se constituye por dos grupos de filiales, de acuerdo a la mayor o menor intensidad de utilización de los STAR y etiquetados como Índice STAR “Bajo" y “Alto", respectivamente. En lo que respecta a la variable Contexto Cultural, hacemos lo propio. Agrupamos los países de las filiales en países pertenecientes al grupo de bajo o alto grado de puntuación en cada dimensión. Esta dicotomización de los países lo efectuamos a partir de puntos de corte teóricamente significativos, para lo cual nos fundamentamos en los análisis aportados por Hofstede (1984, 2001) y Ronen y Shenkar (1985).

Tabla 3

Agrupación de los países de las filiales para cada dimensión cultural

\begin{tabular}{|c|c|c|c|c|}
\hline & Individuatieme & Diatancia al Podter & Averabin a la Incertidumbre & Masculinidad \\
\hline AIEMANIA & 67 & 36 & 65 & 66 \\
\hline ARGIENTINA & 46 & 49 & 86 & 56 \\
\hline nessiL. & $3 \mathbf{s}$ & $\omega$ & 76 & 49 \\
\hline COLOMBA & 64 & 67 & no & 13 \\
\hline REP. CHKCA & 58 & 57 & 74 & 57 \\
\hline CHILE & 23 & 63 & 86 & 28 \\
\hline CHIVA & 20 & 80 & 30 & 66 \\
\hline EREU & 91 & 40 & 46 & 62 \\
\hline Venscas & 71 & 68 & B6 & 43 \\
\hline BEIVO UNMG & so & 35 & 35 & 66 \\
\hline IRI_ANDA & 70 & 28 & 35 & 68 \\
\hline THLA & 76 & 50 & 75 & 70 \\
\hline MEXICO & 30 & 81 & 82 & 69 \\
\hline PORTUGAL. & 27 & 63 & 104 & 45 \\
\hline TURQUAA & 37 & 66 & B5 & 45 \\
\hline VENERUELA & 12 & 81 & 76 & 73 \\
\hline punto de corle & Baje $<-64$ & Bajo $<=50$ & Bajo $<-65$ & baje $<-57$ \\
\hline
\end{tabular}

Las cifras en negrita indican que el país en cuestión pertenece al grupo de "bajo" grado de puntuación en cada una de las dimensiones culturales. Puntuaciones extraídas de Hofstede (1984, 2001).

Fuente: elaboración propia a partir de Hofstede (1984, 2001) y Ronen y Shenkar (1985).

El valor medio de la variable dependiente, Rendimiento, y el número de casos correspondientes para los grupos constituidos figuran a continuación: 
Figura 3

Información complementaria
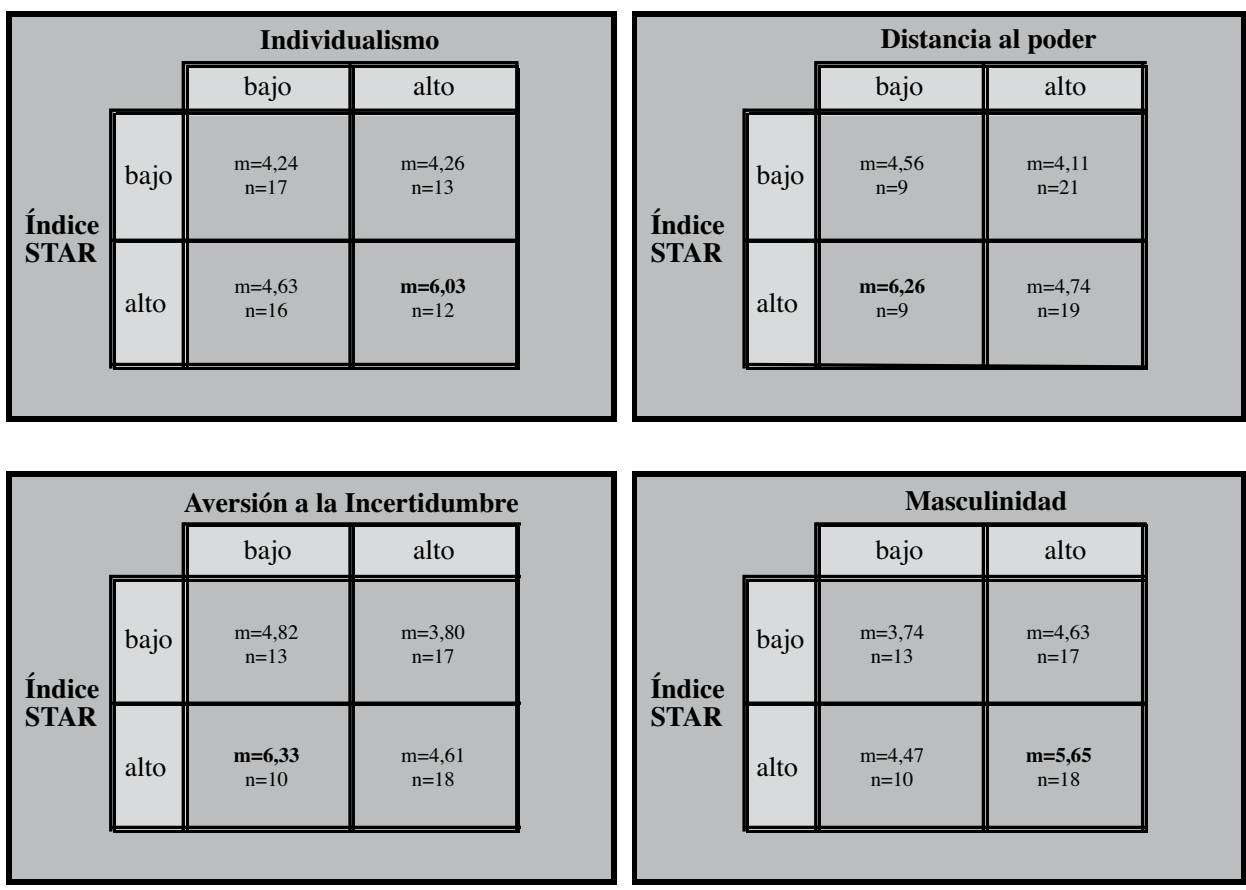

$\mathrm{m}$ : media de rendimientos; $\mathrm{n}: \mathrm{n}^{\mathrm{o}}$ de observaciones

En negrita lo valores más altos registrados

De la información de arriba se deduce que aquellas empresas que pertenecen al grupo de más alto grado en el uso de los STAR alcanzan, en promedio, mejores resultados, algo que ya se ha observado mediante el análisis de regresión anterior. En lo que respecta a la influencia de las dimensiones culturales, dado que los mejores resultados se obtienen en los grupos que se señalarían de acuerdo a la teoría, en principio no sería descabellado pensar que existe un efecto moderador del contexto cultural. Teóricamente, para el caso del Individualismo y la Masculinidad, para los que se estima que interaccionan positivamente con usos más intensivos de estos sistemas, efectivamente, el rendimiento más alto se logra en filiales localizadas en países que puntúan alto en estas dimensiones y en el grado de empleo de los STAR. Lo contrario sucede en el caso de la Distancia al poder y la Aversión a la incertidumbre, pues teóricamente, aquellas compañías que los emplean de forma intensiva y que además se ubican en entornos caracterizados por baja puntuación en cada una de ellas, serían las de mejores resultados empresariales. No obstante, para evaluar estadisticamente la significatividad de las diferencias aplicamos las Pruebas $t$. Las muestras son aleatorias, independientes y siguen una distribución normal (K-S: z=0,986; Sig.=0,285). Los resultados se muestran abajo: 
Tabla 4

Resultados de las Pruebas $t$

\begin{tabular}{|c|c|c|c|}
\hline \multirow[b]{2}{*}{ Dimensién cultural } & Arueba de Levene & \multicolumn{2}{|r|}{ Prected } \\
\hline & $\mathbf{F}$ & $t$ & Diferencia demedias \\
\hline Individualiumo & & & \\
\hline Bajo & ,076 &., 843 &,- 390 \\
\hline Alto & .224 & $-3,563 * 4$ & $-1, m$ \\
\hline Distandia al Poda & & & \\
\hline Bajo & .773 & $-3.219^{\circ *}$ & -1.704 \\
\hline Alto & .000 & $-1,478$ &,- 606 \\
\hline Avervín a la lncertidambre & & & \\
\hline Ihajo & ,057 & $-3,614 * *$ & $-1,513$ \\
\hline Alto & .020 & $-1, \times 31$ & -.927 \\
\hline Nasuculinidad & & & \\
\hline Bajo & 200 & $-1,296$ &,- 723 \\
\hline Alto & .023 & $-2.406^{*}$ & $-1,021$ \\
\hline
\end{tabular}

Variable dependiente: Rendimiento.

La diferencia de medias se calcula restando a1 valor de la media de la variable dependiente para e1 grupo Índice STAR "Bajo”, el valor de la media del grupo Índice STAR “alto".

$* \mathrm{p}<0.05 ; * * \mathrm{p}<0.01 ; * * * \mathrm{p}<0.001$

Los resultados obtenidos sugieren varias cosas:

a) Atendiendo al signo de las diferencias de medias se observa que, independientemente de la dimensión cultural, en todos los casos, los resultados alcanzados por las empresas con un nivel más alto en el uso de los STAR, en promedio, son mejores que los de aquellas otras con nivel inferior. Esto concuerda plenamente con la aceptación de la primera hipótesis.

Teniendo en cuenta la escala de medida empleada para la medición de la variable Rendimiento (desde 1= "Satisfacción muy baja" hasta 7="Satisfacción muy alta"), en algunos casos la divergencia en el rendimiento es relativamente significativa, al alcanzar cerca de dos puntos de diferencia, como sucede en los grupos Individualismo "Alto", Distancia al Poder "Bajo" y en menor medida, en el de Aversión a la Incertidumbre "Bajo". Salvo en el Individualismo "Bajo", donde la diferencia es escasa, en el resto de las situaciones analizadas se estiman diferencias cercanas al punto.

b) Se aprecia claramente que en todas las dimensiones culturales existen diferencias, pero que éstas son solo significativas en el caso de un solo grupo: "alto" o "bajo". La naturaleza y la significatividad de estas discrepancias en el rendimiento se producen en el sentido teorizado en la formulación de las hipótesis.

c) En el caso del Individualismo, en ambos grupos (bajo y alto), la diferencia es a favor del colectivo de empresas que emplean más intensamente las prácticas referidas, pero sólo es significativo en el caso del grupo de empresas en países con una puntuación alta en individualismo. Por consiguiente, la utilización de estos sistemas contribuye de forma positiva en el rendimiento, y a ello se añade que, comparativamente, el diferencial en el rendimiento es más importante en compañías en entornos culturales más individualistas, tal como se presumía de acuerdo a la literatura 
revisada. En otras palabras, se estima que el grado de competitividad de los STAR es comparativa y significativamente mejor en contextos más individualistas. Este resultado concuerda con la evidencia aportada por Bae y Lawler (2000) y GómezMejía et al. (1997) y también con la obtenida por Newman y Nollenn (1996), en este último caso en lo que respecta a la participación de los trabajadores, uno de los componentes de los STAR.

d) Podemos apreciar un comportamiento idéntico en el caso de la dimensión Masculinidad. En contextos culturales con valores más masculinos, estos sistemas alcanzan cotas de diferencial de rendimiento significativamente mejores, en comparación con entornos menos masculinos. Este comportamiento es consistente con los resultados de la investigación de Newman y Nollen (1996), que vinculan el rendimiento positivamente con la aplicación de la retribución contingente (otro componente) en países más masculinos.

e) En cuanto a las dimensiones Distancia al Poder y Aversión a la Incertidumbre, que como se desprende de la literatura mantienen teóricamente una relación de signo negativo con la competitividad de estas prácticas de trabajo, se llega a conclusiones análogas. En ambos casos, cuanto más se emplean éstas, mejores son los resultados empresariales, pero son significativamente mejores sólo en sociedades menos jerárquicas y de mejor aceptación de la incertidumbre, respectivamente. Esto es consistente con los resultados obtenidos por Aycan et al. (2000) y Gómez-Mejía et al. (1997).

A tenor de estos resultados, la información muestral nos refleja un comportamiento que podría reflejar una influencia moderadora de la variable Contexto Cultural. Este hecho parece indicar que el contexto cultural podría actuar como un factor que modera la relación entre el nivel de utilización de las "mejores prácticas” y el rendimiento de la empresa.

\section{CONCLUSIONES Y FUTURAS LÍNEAS DE INVESTIGACIÓN}

Los resultados empíricos sugieren que aquellas empresas que exhiben una mayor intensidad en el empleo de los STAR reportan sensiblemente mejores niveles de rendimiento, independientemente del país de localización.

En cuanto al carácter moderador del contexto cultural, los resultados parecen indicar que el contexto cultural podría actuar como un factor contingente en el efecto positivo que ejerce la implementación de los STAR en el rendimiento empresarial. Sin embargo, dadas las limitaciones del estudio empírico, referidas en el apartado metodológico, el contraste definitivo de esta hipótesis requiriría del empleo de muestras mayores, recogidas de poblaciones diferentes. El reducido tamaño de la muestra, que nos obliga a trabajar con un error muestral relativamente elevado, recomienda considerar los resultados y las conclusiones que hemos alcanzado sólo para esta muestra.

Aunque en muchos casos no sea factible, también sería conveniente para las futuras investigaciones trabajar con entrevistas realizadas a un único sujeto, es decir, el directorgerente o el director corporativo de recursos humanos, para evitar posibles sesgos en la información.

Teniendo en cuenta las limitaciones descritas, estos resultados ponen de manifiesto que las empresas multinacionales deberían, por tanto, favorecer la estandarización de STAR 
en sus filiales, lo que en el futuro puede conducir a una convergencia de las prácticas de gestión en cualquier país del mundo hacia estas prácticas ideales, tal como auguran los postulados universalistas.

Sin embargo, teniendo en cuenta que un contexto cultural favorable contribuye a acrecentar su efectividad, mientras que su ausencia restringe su carácter dinamizador, las empresas deben considerar en todo momento el encaje con los valores culturales del país de localización, al menos en lo que concierne al diseño de las prácticas más específicas del sistema y al esfuerzo que merece la pena realizar en la implantación efectiva de las diferentes prácticas, según la caracterísicas de la cultura de acogida. No todas las prácticas tienen igual influencia en el rendimiento, en función del contexto cultural y, por tanto, el esfuerzo a realizar en su transmisión y implantación debe ser diferente en función de la práctica y el país de la filial.

No obstante, es necesario obtener resultados más concluyentes. Investigaciones futuras deben profundizar en esta línea, con distintas técnicas y con muestras más grandes. Es también necesario ampliar el estudio de la influencia que tienen en el rendimiento las distintas prácticas, en función de las distintas dimensiones de la cultura del país de acogida.

Otra línea interesante de estudio es cambiar el enfoque en la operacionalización del contexto cultural. Nosotros hemos recurrido al modelo de Hofstede, puesto que es uno de los pocos modelos que es cuantitativo y contempla un amplio conjunto de países, pero adolece de la limitación que suele implicar el uso de constructos teóricos en la operacionalización de las variables complejas, como es claramente el caso de esta variable. Además de las críticas de naturaleza técnica que se le pueden hacer (McSweeney, 2002), hoy en día, por la cambiante realidad demográfica y social en la que vivimos, cada vez un mayor número de autores constatan la necesidad de ajustar los parámetros de medición donde se sustentan los modelos culturales actuales (Ardichvili y Gasparishvili, 2001; Child, 2002; Dolan et al., 2004; Edwards y Rees, 2006; Gerhart, 2008; Peppas, 2001; Sackmann y Phillips, 2004).

\section{REFERENCIAS BIBLIOGRÁFICAS}

ADLER, N.J. (2002): International Dimensions of Organizational Behavior, Boston, South-Western publishing.

AGARWAL, S. (1994): 'Socio-Cultural Distance and the Choice of Joint Ventures: A Contingency Perspective', Journal of International Marketing, Vol. 2, No. 2, pp. 63-80.

AGUINIS, H. (1995): 'Statistical power problems with Moderated Multiple Regression in management research', Journal of Management, Vol. 21, No. 6, pp. 1141-1158.

AHMAD, S. Y SCHROEDER, R.G. (2003): 'The impact of human resource management practices on operational performance: recognizing country and industry differences', Journal of Operations Management, Vol. 21, No. 1, pp. 19-43.

AIKEN, L.S. Y WEST, S.G. (1991): Multiple Regression: Testing and Interpreting Interactions, Thousand Oaks, California, Sage.

APPELBAUM, E.; BAILEY, T.; BERG, P. Y KALLEBERG, A.L. (2000): Manufacturing Advantage: Why High-Performance Work Systems Pay Off, Londres, Cornell University Press.

ARDICHVILI, A. Y GASPARISHVILI, A. (2003): 'Russian and Georgian entrepreneurs and nonentrepreneurs: A study of Value Differences', Organization Studies, Vol. 24, No. 1, pp. 29-46.

ARTHUR, J.B. (1994): 'Effects of human resource systems on manufacturing performance and turnover', Academy of Management Journal, Vol. 37, No. 3, pp. 670-687. 
AYCAN, Z. ; KANUNGO, R.N. ; MENDONCA, M. ; YU, K., DELLER, J. ; STAHL, G. Y KURSHID, A. (2000): 'Impact of Culture on Human Resource Management Practices. A 10 Country Comparison', Applied Psychology: An International Review, Vol. 49, No. 1, pp. 192-221.

BAE, J. ; CHEN, S. ; WAN, T.W.D. ; LAWLER, J.J. Y WALUMBWA, F.O. (2003): 'Human resource strategy and firm performance in Pacific Rim countries', International Journal of $\mathrm{Hu}$ man Resource Management, Vol. 14, No. 8, pp. 1308-1332.

BAE, J. Y ROWLEY, C. (2001): 'The impact of Globalization on HRM: The Case of South Korea', Journal of World Business, Vol. 36, No. 4, pp. 402-428.

BAE, J. Y LAWLER, J.J. (2000): 'Organizational and HRM strategies in Korea: impact on firm performance in an emerging economy', Academy of Management Journal, Vol. 43, No. 3, pp. 502-517.

BAE, J. ; CHEN, S. Y LAWLER, J. (1998): 'Variations in human resource management in Asian countries: MNC home-country and host-country effects', International Journal of Human Resource Management, Vol. 9, No. 4, pp. 653-670.

BARNEY, J.B. Y WRIGHT, P.M. (1998): 'On Becoming a Strategic Partner: The Role of Human Resources in Gaining Competitive Advantage', Human Resource Management, Vol. 37, No. 1, pp. 31-46.

BECKER, B.E. ; HUSELID, M.A. ; PICKUS, P.S. Y SPRATT, M.F. (1997): 'HR as a source of shareholder value: research and recommendations', Human Resource Management, primavera, Vol. 36, No. 1 , pp. 39-47.

BJÖRKMAN, I. Y XIUCHENG, F. (2002): 'Human resource management and the performance of Western firms in China', International Journal of Human Resource Management, Vol. 13, No. 6, pp. 853-864.

BLACK, B. (1999): 'National culture and high commitment management', Employee Relations, Vol. 21, No. 4, pp. 389-404.

BONACHE, J. Y CERVIÑO, J. (1997): "La adaptación cultural en la empresa multinacional: el conflicto entre la teoría y la práctica”, Información Comercial Española, Ministerio de Economía y Hacienda, No. 761, pp. 33-48.

BORMAN, W.C. (1991): 'Job behaviour, performance and effectiveness', en Handbook of Industrial and Organizational Psychology, ed. M.D. Dunnette. y L.M. Hough, Palo Alto: Consulting Psychology Press, pp. 271-326.

BOSELIE, P.; PAAUWE, J Y JANSEN, P. (2001): 'Human resource management and performance: lessons from the Netherlands', International Journal of Human Resource Management, Vol. 12, No. 7, pp. 1107-1125.

BOXALL, P. Y PURCELL, J. (2003): Strategy and Human Resource Management, Hampshire, Palgrave MacMillan.

CAMELO, C. ; MARTÍN, F. ; ROMERO, P.M. Y VALLE, R. (2004): 'Human resources management in Spain: is it posible to speak of a typical model?', International Journal of Human Resource Management, Vol. 15, No. 6, pp. 935-958.

CAPPELLI, P. Y NEUMARK, D. (2001): 'Do "high-performance" work practices improve establishment-level outcomes?', Industrial and Labor Relations Review, Vol. 54, No. 4, pp. 737-775.

CHANG, E. (2006): 'Individual pay for performance and commitment HR practices in South Korea', Journal of World Business, Vol. 41, No. 4, pp. 368-381.

CHIANG, F. (2005): 'A critical examination of Hofstede's thesis and its application to international reward management', International Journal of Human Resource Management, Vol. 16, No. 9; pp. 1545-1563.

CHILD, J. (2002): 'Theorizing about organization cross-nationally: part 2 - towards a synthesis', en Managing Across Cultures: Issues and Perspectives, ed. M. Warner. y P. Joynt, London: Thompson, pp. 40-56.

COHEN, J. Y COHEN, P. (1983): Applied Multiple Regression/Correlation Analysis for the Behavioral Sciences, (2nd. ed.), Hillsdale, New York, Lawrence Erlbaum Associates. 
DELANEY, J.T. Y HUSELID, M.A. (1996): 'The impact of human resource management practices on perceptions of organizational performance', Academy of Management Journal, Vol. 39, No. 4, pp. 949-969.

DELERY, J.E. (1998): 'Issues of Fit in Strategic Human Resource Management: Implications for research', Human Resource Management Review, Vol. 8, No. 3, pp. 289-309.

DELERY, J.E. Y DOTY, D.H. (1996): 'Modes of theorizing in strategic human resource management: tests of universalistic, contingency and configurational performance predictions', Academy of Management Journal, Vol. 39, No. 4, pp. 802-835.

DOLAN, S.L. ; MARTÍN-PRIUS, A. ; DÍEZ-PIÑOL, M. ; MARTÍNEZ-FIERRO, S. Y FERNÁNDEZ-ALLES, M. (2004): 'Exploratory Study of of Within-Country Differences in Work and Life Values', International Journal of Cross Cultural Management, Vol. 4, No. 2, pp. 157-180.

EBY, L.T. ; ADAMS, D.M. ; RUSSELL, J.E.A. ; GABY, S.H. (2000): 'Perceptions of organizational readiness for change: factors related to employees' reactions to the implementation of teambased selling', Human Relations, Vol. 53, No. 1, pp. 419-442.

EDWARDS, T. Y REES, C. (2006): International Human Resource Management. Globalization, National Systems and Multinational Companies, Essex, England, Pearson Education.

FEY, C.F. Y BJÖRKMAN, I. (2001): 'The Effect of Human Resource Management Practices on MNC Subsidiary Performance in Russia', Journal of International Business Studies, Vol. 32, No. 1, pp. 59-75.

GARCÍA, A.M. Y GARCÍA, M.G. (2007): "Diferencias culturales y comportamiento emprendedor: un análisis cross-cultural intrapaís", Revista Europea de Dirección y Economía de la Empresa, Vol. 16, No. 4, pp.25-46.

GEARY, J.K. (1999). 'The new workplace: change at work in Ireland', International Journal of Human Resource Management, Vol. 10, No. 5, pp. 870-890.

GERHART, B. (2008): 'Cross Cultural Management Research', International Journal of Cross Cultural Research, Vol. 8, No. 3, pp. 259-274.

GERHART, B. Y FANG, M. (2005): 'National culture and human resource management: assumptions and Evidence', International Journal of Human Resource Management, Vol. 16, No. 6, pp. 971- 986.

GERHART, B. Y MILKOVICH, G.T. (1990): 'Organizational differences in managerial compensation and financial performance', Academy of Management Journal, Vol. 33, No. 4, pp. 663-691.

GILL, R. Y WONG, A. (1998): 'The cross-cultural transfer of management practices: The case of Japanese human resource management practices in Singapore', International Journal of Human Resource Management, Vol. 9, No. 1, pp. 116-135.

GODARD, J. (2004): 'A Critical Assessment of the High-Performance Paradigm', British Journal of Industrial Relations, Vol. 42, No. 2, pp. 349-378.

GÓMEZ-MEJÍA, L.R. Y PALICH, L.E. (1997): 'Cultural diversity and the performance of multinational firms', Journal of International Business Studies, Vol. 28, 2 No., pp. 309-335.

GÓMEZ-MEJÍA, L.R.; BALKIN, D.B. Y CARDY, R. (1997): Human Resource Management, Madrid, Prentice Hall.

GOODERHAM, P. ; PARRY, E. Y RINGDAL, K. (2008): 'The impact of bundles of strategic human resource management practices on the performance of European firms', International Journal of Human Resource Management, Vol. 19, No. 11, pp. 2041-2056.

GUEST, D.E. ; MICHIE, J. ; CONWAY, N. Y SHEEHAN, M. (2003): 'Human Resource Management and Corporate Performance in the UK', British Journal of Industrial Relations, Vol. 41, No. 2, pp. 291-314.

GUEST, D.E. (1997): 'Human resource management and performance: a review and research agenda', International Journal of Human Resource Management, Vol. 8, No. 3, pp. 263-276. 
GUTHRIE, J.P. ; FLOOD, P.C. ; LIU, W. Y MACCURTAIN, S. (2009): 'High performance work systems in Ireland: human resource and organizational outcomes', International Journal of Human Resource Management, Vol. 20, No. 1, pp. 112-125.

GUTHRIE, J.P. (2001): 'High-involvement work practices, turnover, and productivity: evidence from New Zealand', Academy of Management Journal, Vol. 44, No. 1, pp. 180-190.

HAIR, J.F. ; ANDERSON, R.E. ; TATHAM, R.L. Y BLACK, W.C. (1999), Análisis Multivariante, ( $5^{\mathrm{a}}$ ed.), Madrid, Pearson Educación.

HOFSTEDE, G. (2001): Culture's consecuences: Comparing Values, Behaviors, Institutions and Organizations Across Nations, Londres, Sage.

HOFSTEDE, G. (1997): Culture and Organizations: software of the mind, New York, MacGrawHill.

HOFSTEDE, G. (1984): Culture's Consecuences. International differences in work-related values. California, Sage.

HOQUE, K. (1999): 'Human Resource Management and Performance in the UK Hotel Industry', British Journal of Industrial Relations, Vol. 37, No. 3, pp. 419-443.

HUSELID, M.A. (1995): 'The impact of human resource management practices on turnover, productivity, and corporate financial performance', Academy of Management Journal, Vol. 38, pp.635-672.

ICHNIOWSKI, C. ; SHAW, K. Y PRENNUSHI, G. (1997): 'The effects of human resource management practices on productivity: A study of steel finishing lines', The American Economic Review, Vol. 87, No. 3, pp. 291-313.

JACCARD, J. ; TURRISI, R. Y WAN, C.K. (1990): 'Interaction Effects in Multiple Regression', Sage University Paper Series on Quantitative Applications in the Social Sciences, 72, Newbury Park, California, Sage.

JACKSON, S.E. Y SCHULER, R.S. (1995): 'Understanding human resource management in the context of organizations and their environments", Annual Review of Psychology, Vol. 46, pp. 237- 264.

KIM, Y. Y GRAY, S.J. (2005): 'Strategic factors influencing international human resource management practices: an empirical study of Australian multinational corporations', International Journal of Human Resource Management, Vol. 16, No. 5, pp. 809-830.

KIRKMAN, B.L. Y SHAPIRO, D.I. (2001): 'The impact of cultural values on job satisfaction and organizational commitment in self-managing work teams: the mediating role of employee resistance', Academy of Management Journal, Vol. 44, No. 3, pp. 557-569.

KOCH, M.J. Y MCGRATH, R.G. (1996): 'Improving labor productivity: human resource management policies do matter', Strategic Management Journal, Vol. 17, No. 4, pp. 335-354.

LAURENT, A. (1986): 'The cross-cultural puzzle of international human resource management. International Human Resource Management: A field in infancy', Human Resource Management, Vol. 25, No. 1, pp. 91-102.

LAWLER, J.J. ; CHEN, S. Y BAE, J. (2000): 'Scale of operations, Human Resource Systems and Firm Performance in East and Southeast Asia', Research and Practice in Human Resource Management, Vol. 8, No. 1, pp. 3-20.

LÓPEZ, C. (2007): "Evolución de la inversión directa exterior de la gran empresa española, 19882003. El papel de las empresas cotizadas", Tribuna de Economía de Información Comercial Española, No. 836, mayo-junio, pp. pp. 107-125.

MACDUFFIE, J.P. (1995): 'Human resource bundles and manufacturing performance: Organizational logic and flexible production systems in the world auto industry', Industrial and Labor Relations Review, Vol. 48, No. 2, pp. 197-221.

MCSWEENEY, B. (2002): 'Hofstede's model of national culture differences and their consequences: A triumph of faith-a failure of analyses', Human relations, Vol. 55, No. 1, pp. 89-118.

MEAD, R. (1998): International Management. Cross cultural dimensions, Oxford, Blackwell. 
MOROSINI, P. ; SHANE, S. Y SINGH, H. (1998): 'National cultural distance and cross border adquisition performance', Journal of International Business Studies, Vol. 29, No. 1, pp. 137-158.

MYLONI, B. ; HARZING, A.W. Y MIRZA, H. (2004): 'Host country specific factors and the transfer of Human Resource Management practices in Multinational companies', International Journal of Manpower, Vol. 25, No. 6, pp. 518-534.

NEWMAN, K. Y NOLLEN, S. (1996): 'Culture and Congruence: The fit between management practices and national culture', Journal of International Business Studies, Vol. 27, No. 4, pp. 753-779.

NGO, H.Y. ; TURBAN, D. ; LAU, C.M. Y LUI, S.Y. (1998): 'Human resource practices and firm performance of multinational corporations: influences of country origin', International Journal of Human Resource Management, Vol. 9, No. 4, pp. 632-652.

OSTERMAN, P. (1994): 'How common is workplace transformation and who adopts it?', Industrial and Labor Relations Review, Vol. 47, No. 2, pp. 173-188.

PARK, H.J. ; MITSUHASHI, H. ; FEY, C. F. Y BJÖRKMAN, I. (2003): 'The effect of human resource management practices on Japanese MNC subsidiary performance: a partial mediating model', International Journal of Human Resource Management, Vol. 14, No. 8, pp. 1391-1406.

PECK, S.R. (1994): 'Exploring the link between organizational strategy and the employment relationship: the role of human resources policies', Journal of Management Studies, Vol. 31, No. 5, pp. 715-736.

PEPPAS, S.C. (2001): 'Subcultural Simmilarities and Differences: An examination of US Core Values', Cross Cultural Management, Vol. 8, No. 1, pp. 59-70.

PFEFFER, J. (1998): Human Equation. Building profits by putting people first, Boston, MA, Harvard Business School Press.

RHODES, J. ; WALSH, P. Y LOK, P.(2008): 'Convergence and divergence issues in strategic management - Indonesia's experience with the Balanced Scorecard in HR management', International Journal of Human Resource Management, Vol. 19, No. 6, pp. 1170-1185.

RODRÍGUEZ, J.M. Y VENTURA, J. (2003): 'Human resource management systems and organizational performance: an analysis of the Spanish manufacturing industry', International Journal of Human Resource Management, Vol. 14, No. 7, pp. 1206-1226.

RONEN, S. Y SHENKAR, O. (1985): 'Clustering Countries on Attitudinal Dimensions: A Review and Synthesis', Academy of Management Review, Vol. 10, No. 3, pp. 435-454.

RUSELL, C. G. ; TERBORG, J.R. Y POWERS, M.L. (1985): 'Organizational performance and organizational level training and support', Personnel Psychology, Vol. 38, No. 4, pp. 849-863.

SACKMANN, S.A. Y PHILLIPS, M.E. (2004): 'Contextual Influences on Culture Research. Shifting Assumptions for New Workplace Realities', International Journal of Cross Cultural Management, Vol. 4, No. 3, pp. 370-390.

SANZ-VALLE, R. ; SABATER-SÁNCHEZ, R. Y ARAGÓN-SÁNCHEZ, A. (1999): 'Human resource management and business strategy links: an empirical study', International Journal of Human Resource Management, Vol. 10, No. 4, pp. 655-671.

SCHULER, R.S. Y ROGOVSKY, N. (1998): 'Understanding Compensation Practice Variations Across Firms: The Impact of National Culture', Journal of International Business Studies, Vol. 29, No. 1, pp. 159-177.

STEENSMA, H.K. ; MARINO, L. ; WEAVER, K.M. Y DICKSON, P.H. (2000) 'The influence of national culture on the formation of technology alliances by entrepreneurial firms', Academy of Management Journal, Vol. 43, No. 5, pp. 951-973.

SØNDERGAARD, M. (1994). 'Research Note: Hofstede's Consecuences: A Study os Reviews, Citations, and Replications', Organization Studies, Vol. 15, No. 3, pp. 447-456.

TAYEB, M.H. (2005): International Human Resource Management. A multinational Company Perspective, Oxford, Oxford University Press.

TEPSTRA, D.E. Y ROZELL, E. (1993): 'The relationship of staffing practices to organizational level measures of performance', Personnel Psychology, Vol. 46, pp. 27-48. 
TROMPENAARS, F. (1994): Riding the Waves of culture. Understanding cultural diversity in business, Londres, Nicholas Brealey.

VERBURG, R.M. ; DRENTH, P.J.D. ; KOOPMAN, P.L. ; VAN MUIJEN, J.J. Y WANG, Z. (1999): 'Managing human resources across cultures: a comparative analyses of practices in industrial enterprises in China and The Netherlands', International Journal of Human Resource Management, Vol. 10, No. 3, pp. 391-410.

WAY, S.A. (2002): 'High Performance Work Systems and Intermediate Indicators of Firm Performance within the US Small Business Sector', Journal of Management, Vol. 28, No. 6, pp. 765-785.

WOOD, S. Y MENEZES, L. (1998): 'High commitment management in UK: evidence from the workplace industrial relations survey and employers' manpower and skills practices survey', Human Relations, Vol. 51, No. 4, pp. 485-515.

YOUNDT, M.A. ; SNELL, S.A. ; DEAN, J.W. JR. Y LEPAK, D.P. (1996): 'Human resource management, manufacturing strategy and firm performance', Academy of Management Journal, Vol. 39, No. 4, pp. 836-866.

YUEN, E.C. Y KEE, H.T. (1993): 'Headquarters, host-culture and organizational influences on HRM policies and practices', Management International Review, Vol. 33, No. 4, pp. 361-380. 